\title{
Possible Evidence for Free Precession of a Strongly Magnetized Neutron Star in the Magnetar 4U 0142+61
}

\author{
K. Makishima,,${ }^{1,2,3}$ T. Enoto, ${ }^{4,5}$ J. S. Hiraga, ${ }^{2}$ T. Nakano, ${ }^{1}$ K. Nakazawa, ${ }^{1}$ S. Sakurai, ${ }^{1}$ M. Sasano, ${ }^{1}$ and H. Murakami ${ }^{1}$ \\ ${ }^{1}$ Department of Physics, Graduate School of Science, \\ the University of Tokyo, 7-3-1 Hongo, Bunkyo-ku, Tokyo 113-0033, Japan \\ ${ }^{2}$ Research Center for the Early Universe, Graduate School of Science, \\ the University of Tokyo, 7-3-1 Hongo, Bunkyo-ku, Tokyo 113-0033, Japan \\ ${ }^{3}$ MAXI team, RIKEN, 2-1 Hirosawa, Wako, Saitama 351-0198, Japan \\ ${ }^{4}$ High Energy Astrophysics Laboratory, RIKEN Nishina Center, 2-1 Hirosawa, Wako, Saitama 351-0198, Japan \\ ${ }^{5}$ NASA Goddard Space Flight Center, Astrophysics Science Division, Code 662, Greenbelt, MD 20771, USA
}

(Dated: September 11, 2018)

\begin{abstract}
Magnetars are a special type of neutron stars, considered to have extreme dipole magnetic fields reaching $\sim 10^{11} \mathrm{~T}$. The magnetar $4 \mathrm{U} 0142+61$, one of prototypes of this class, was studied in broadband X-rays (0.5-70 keV) with the Suzaku observatory. In hard X-rays (15-40 keV), its 8.69 sec pulsations suffered slow phase modulations by $\pm 0.7 \mathrm{sec}$, with a period of $\sim 15$ hours. When this effect is interpreted as free precession of the neutron star, the object is inferred to deviate from spherical symmetry by $\sim 1.6 \times 10^{-4}$ in its moments of inertia. This deformation, when ascribed to magnetic pressure, suggests a strong toroidal magnetic field, $\sim 10^{12} \mathrm{~T}$, residing inside the object. This provides one of the first observational approaches towards toroidal magnetic fields of magnetars.
\end{abstract}

PACS numbers: 97.60.Jd, 97.80.Jp, 97.10.Ld, 45.20.D-

Inroduction.- Neutron stars (NSs) are deemed to possess strong magnetic field (MF) of $10^{4}-10^{11} \mathrm{~T}[1] 3$. Their MF is attributed to, e.g., proton superfluids [3], or ferromagnetism in nuclear matter [4, 5], but without clear consensus. When studying their magnetism, a subclass of importance is magnetars [3, 6, 7], isolated NSs believed to have extreme dipole MFs of $B_{\mathrm{d}}=10^{10}-10^{11}$ T. Their persistent and burst-like X-rays are thought to be powered by the MF energy, because their luminosity much exceeds the rate of their rotational energy loss.

We expect magnetars to harbor even stronger toroidal $\mathrm{MF}, B_{\mathrm{t}}[3,6,6,8,[9]$, because differential rotation in their progenitors will tightly wind up the MF lines during their final collapse. We then expect some of the internal MF lines to emerge from the stellar surface [9], to form multipoles therein. These expectations are supported by the recently discovered low- $B_{\mathrm{d}}$ magnetar, SGR 0418+5729 [10], because its burst activity would require MFs exceeding the measured $B_{\mathrm{d}}=6 \times 10^{8} \mathrm{~T}$, and it shows spectral evidence for much stronger multipole surface MF [11]. However, more direct estimates of $B_{\mathrm{t}}$ remained difficult.

$\mathrm{X}$-ray spectra of magnetars ubiquitously consist of a black-body-like soft component and a distinct hard X-ray tail [12, 13], dominant in energies below and above $\sim 10$ $\mathrm{keV}$, respectively, both pulsed strongly at the NS's rotation period. While the former must be thermal emission from two magnetic poles, the latter may be non-thermal photons from possibly different regions on or around the NS [13]. The behavior of the two components will thus provide clues to the magnetic structure of magnetars.

We conducted accordingly two observations of $4 \mathrm{U} 0142+61$, one of the X-ray brightest magnetars. It has a rotation period of $8.69 \mathrm{sec}$, and it allowed one of the first detections of the hard component 12, 14, 15]. On the 2nd occasion, its $8.69 \mathrm{sec}$ pulsation in hard X-rays were found to exhibit a slow phase modulation. The effect may be taken as evidence for free precession of this NS, and suggests its magnetic deformation with $B_{\mathrm{t}} \sim 10^{12} \mathrm{~T}$.

Observation. - The two observations of $4 \mathrm{U} 0142+61$ were conducted with a 2 year interval, using the Suzaku X-ray observatory [16]. The soft and hard components of magnetars match ideally with the two Suzaku instruments; the X-ray Imaging Spectrometer (XIS) [17] sensitive in $0.3-10 \mathrm{keV}$, and the Hard X-ray Detector (HXD) [18] working in 10-600 keV. Following the first observation made in 2007 August [19], the 2nd one reported here was performed on 2009 August 12-14, for a gross exposure of $186 \mathrm{ksec}$ (net $102 \mathrm{ksec}$ ). We operated the XIS in 1/4-frame mode and the HXD in normal mode, with a time resolution of $2.0 \mathrm{sec}$ and $61 \mu \mathrm{sec}$, respectively.

The source was detected at background-removed count rates of $6.74 \pm 0.01 \mathrm{c} \mathrm{s}^{-1}$ with the XIS in $0.4-10 \mathrm{keV}$ (per camera), and $(3.08 \pm 0.28) \times 10^{-2} \mathrm{c} \mathrm{s}^{-1}$ with the HXD in $15-70 \mathrm{keV}$; both agree within $\sim 15 \%$ with those in 2007 [19]. Converting each photon arrival time to that at the Solar system barycenter, and analyzing the XIS data via epoch folding analysis, we detected, as shown in Fig. 1 (a), the soft X-ray pulses at a barycentric period of

$$
P_{\text {soft }}=8.68891 \pm 0.00010 \mathrm{sec} .
$$

Together with the folded soft X-ray pulse profile in Fig. 1 (d), this reconfirms the previous measurements [20].

Results. - We also searched the 15-40 keV HXD data for the expected hard X-ray pulsation [12, 15, 19]. Because of lower statistics, we employed the $Z_{n}^{2}$ technique 21] which is free from the event binning ambiguity. If 
no periodicity, the $Z_{n}^{2}$ values should obey a $\chi^{2}$ distribution of $2 n$ degrees of freedom (dof). Since the hard X-ray pulse profile of $4 \mathrm{U} 0142+61$ is double-peaked [12, 15] with possible structurs [19], we tried $n=3$ and 4 .

As given in Fig. 1 (b), the HXD periodograms with $n=3$ and 4 both show a small peak at $\sim 8.689 \mathrm{sec}$, at the error boundary of eq.(1), but its significance is rather low, and higher peaks are seen at different periods. This result was unexpected, as the hard X-ray intensity and the observing time were both similar to those in 2007, wherein the pulses were detected clearly both with the HXD [19] and XIS: some changes must have taken place in the hard component. Specifically, the hard Xray power, originally at eq. (11) (and its harmonics), may have been scattered out over a period range of Fig. 11 by, e.g., some pulse-shape variations as suggested before [19], or more likely, by pulse-phase modulations. We thus came to suspect that the hard X-ray pulses in 2009 suffer, for unspecified reasons, some phase modulations.

We assume that the $8.69 \mathrm{sec} \mathrm{X}$-ray pulsation in the 2009 HXD data is phase-modulated, so that the peak timing $t$ of each pulse shifts by $\Delta t=A \sin (2 \pi t / T-\phi)$, where $T, A$, and $\phi$ are the period, amplitude, and ini-
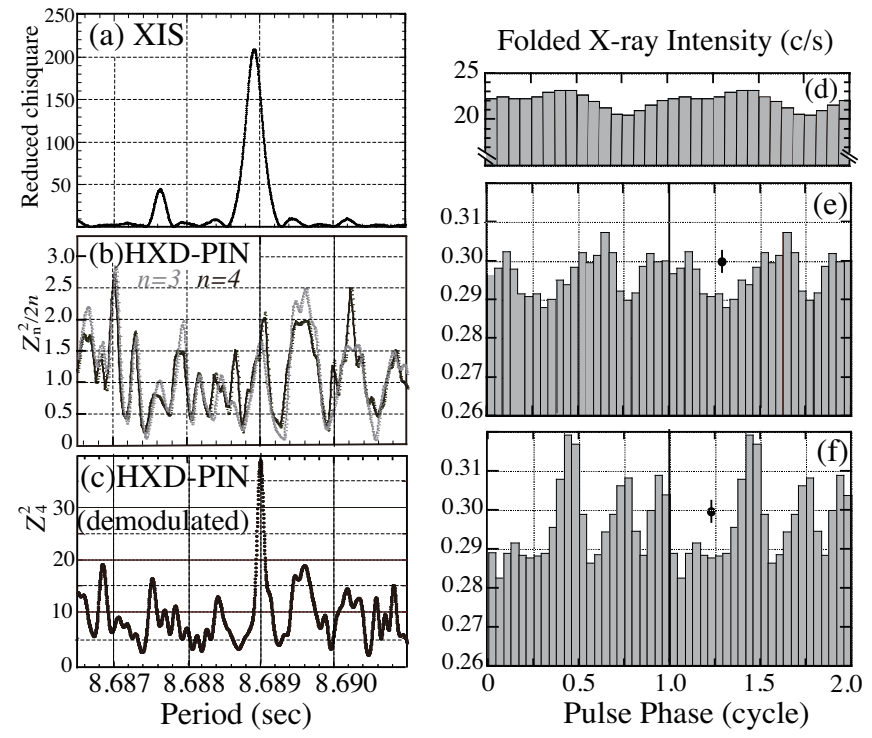

FIG. 1: (a) A 1-10 keV XIS0+XIS1+XIS3 periodogram, calculated via folding analysis using 16 phase bins per cycle. (b) Periodograms from the background-inclusive 15-40 HXDPIN data, calculated using the $Z_{n}^{2}$ technique with $n=3$ (grey) and $n=4$ (black). (c) The same $Z_{4}^{2}$ periodogram as in (b), but after the demodulation correction employing the bestestimate conditions (see text). (d) A soft X-ray pulse profile (two cycles), obtained by folding the 1-10 keV XIS data at eq.(11). (e) The background-inclusive 15-40 keV HXD-PIN data, similarly folded at $P_{\text {hard }}$. A running average over three adjacent bins was applied. The background level corresponds to $0.26 \mathrm{c} \mathrm{s}^{-1}$, and the error bar represents statistical \pm 1 sigma. (f) The same as (e), but after the demodulation procedure. tial phase of the assumed modulation, respectively. Such effects would be removed by shifting the arrival times of individual HXD photons by $-\Delta t$. Employing a trial triplet $(T, A, \phi)$, we applied these time displacements to the HXD data, and re-calculated the $Z_{n}^{2}$ periodograms over an error range of eq.(11) to see whether the pulse significance changes. Then, we searched for the highest pulse significance, by scanning the three parameters over a range of $A=0-1.2 \mathrm{sec}(0.05 \mathrm{sec}$ step $), \phi=0-360^{\circ}$ $\left(3^{\circ}-10^{\circ}\right.$ step $)$, and $T=35-70 \mathrm{ksec}(1-2.5 \mathrm{ksec}$ step $)$. The $Z_{n}^{2}$ harmonic parameter was chosen to be $n=4$.

This "demodulation" analysis has yielded results in Fig. 2. Under a condition of $T=55.0 \mathrm{ksec}$, the pulse significance has increased drastically to $Z_{4}^{2}=39.5$ (panel a) when $\phi=75^{\circ} \pm 30^{\circ}$ (panel b) and $A=0.7 \pm 0.3 \mathrm{sec}$ (panel c) are employed. As in panel (d), the modulation period was constrained as $T=55 \pm 4 \mathrm{ksec}$, where neither background variation nor observing window has significant power. The errors of $\phi, A$, and $T$ are represented by the standard deviations of Gaussians fitted to the distributions (above uniform backgrounds) in Fig. 2(b)-(d). When the data are demodulated with these conditions, the HXD periodogram, Fig.1 (b), changed into Fig.11(c); it reveals a prominent single peak at $P_{\text {hard }}=8.68899(5)$ sec, where the error was determined from the peak width in Fig. 1(c). This is consistent with $P_{\text {soft }}$ within errors.

Figures 1(e) and (f), respectively, show the HXD pulse profiles before and after the demodulation, both folded at $P_{\text {hard }}$. The latter exhibits a significantly larger pulse amplitude and richer fine structures than the former. In addition, the HXD pulse-peak phase has been brought closer to that of the XIS, as in previous observations [15]. We further folded the 2009 HXD data (without demodulation) into 12 bins at $P_{\text {hard }}$, over six separate phases of the $T=55 \mathrm{ksec}$ period, and cross-correlated the profiles with that in Fig. 1(f). The results, given in Fig. [3(a), visualize the sinusoidal nature of the modulation.

Can the peak in Fig. 2 arise by chance when considering the many trials in $T, A, \phi$, and $P$ ? As a "control" study, we repeated, 356 times, the same analysis as Fig. 2 at periods of $P=P_{\text {hard }}+\Delta P$, scanning the offset $\Delta P$ from -0.1 to +0.1 sec with a 0.5 msec step (but avoiding $\Delta P=0$ and side lobes of $\left.P_{\text {hard }}\right)$. Extrapolating the obtained $Z_{4}^{2}$ grand maxima distribution and taking its uncertainty into account, the chance probability to find a value of $Z_{4}^{2} \geq 39.5$ in a search like Fig. 22 was estimated as $p_{\mathrm{z} 2} \equiv(0.8-2.6) \times 10^{-3}$. We also found that individual $Z_{4}^{2}$ values around $P=P_{\text {hard }}$ (but away from $T=55 \mathrm{ksec}$ ) roughly obey a $\chi^{2}$ distribution with 9 dof, instead of 8 , due to the pulsation. We hence multiplied $p_{\mathrm{z} 2}$ by a factor $\Psi_{9}(39.5) / \Psi_{8}(39.5)=9.4 \times 10^{-6} / 4.0 \times 10^{-6}=2.4$, to obtain the overall chance probability of $(1.9-6.2) \times 10^{-3}$, where $\Psi_{\nu}(x)$ is upper integral for a $\chi^{2}$ distribution of $\nu$ dof. Thus, at $>99 \%$ confidence, we can exclude the case where the peak in Fig. 2 arises via chance fluctuations.

For further examination, we applied exactly the same 
demodulation search to three blank-sky HXD data sets, and another for the Crab Nebula representing high countrate signals. However, these data sets all gave $Z_{4}^{2}<30$. Since the implied upper probability integral, $\Psi_{8}(30.0)=$ $2.0 \times 10^{-4}$, is still much larger than $\Psi_{9}(39.5)$, the $55 \mathrm{ksec}$ modulation in $4 \mathrm{U} 0142+61$ is unlikely to be instrumental. We next re-analyzed the $2007 \mathrm{HXD}$ data of $4 \mathrm{U} 0142+61$ with the $Z_{4}^{2}$ method, and reconfirmed the hard X-ray pulsation with a high significance of $Z_{4}^{2}=52.0$, at 8.68878(5) sec as in [19]. The 2007 data were further subjected to the same demodulation search, over a range of $T=55 \pm 10$ ksec which is 2.5 times wider than the 2009 uncertainty. However, the HXD data in 2007 were not very sensitive to $A$ or $T$, yielding a rather loose limit of $A<0.9$. Since this limit overlaps with the error range of $A$ in 2009 , the 2007 HXD data accommodate the hard X-ray modulation, but do not give an independent support to it.

Finally, the same analysis was applied to the two $(2007$ and 2009) XIS data sets of $4 \mathrm{U} 0142+61$. However, the soft X-ray pulses on neither occasion exhibited evidence for phase modulation over $T=55 \pm 10 \mathrm{ksec}$, and the highest pulse significance was obtained at $A_{\sim}^{<0.1}$ sec. Through a simulation, we confirmed that this result is not due to the insufficient time resolution $(2.0 \mathrm{sec})$ of the XIS data: a $55 \mathrm{ksec}$ phase modulation with $A=0.7 \mathrm{sec}$ would have been detected within an error of $\Delta A \sim \pm 0.2$ sec (90\% limits). We thus place an upper limit of $A<0.3$ sec for the soft X-ray pulse-phase modulation at $55 \mathrm{ksec}$.

Discussion.- The pulse-phase variation in the 2009
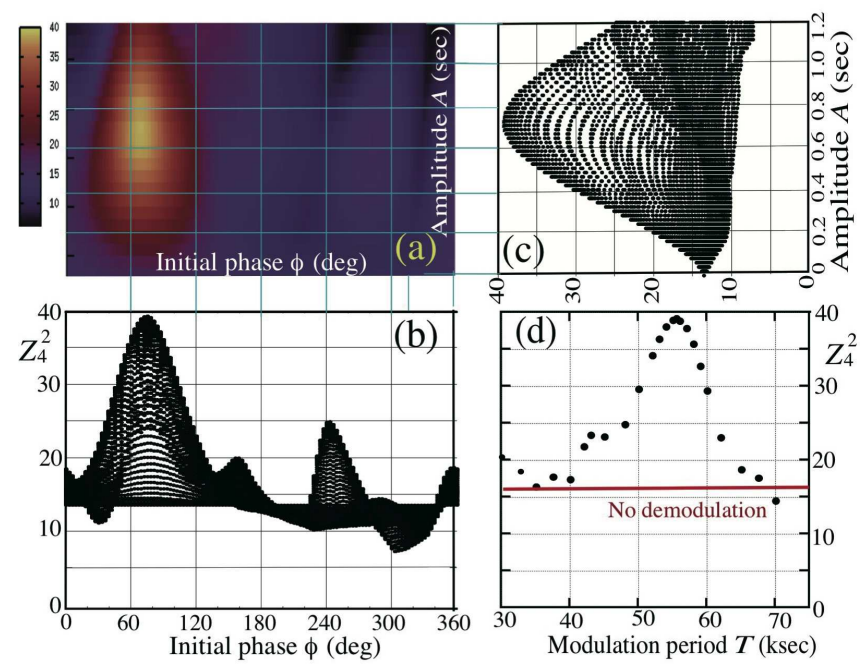

FIG. 2: Results of the $Z_{4}^{2}$ "demodulation" analysis, assuming a periodic phase shift in the 15-40 keV HXD pulses in 2009 . (a) A two-dimensional color map, on the $(\phi, A)$ plane, of the $Z_{4}^{2}$ maximum found over the period range of eq.(1), for $T=$ $55.0 \mathrm{ksec}$. (b) The projection of panel (a) onto the $\phi$-axis, where the vertical data scatter reflects differences in $A$. (c) The same as panel (b), but projected onto the $A$ axis. (d) The maximum values of $Z_{4}^{2}$ found in maps as panel (a), plotted against $T$. (a)

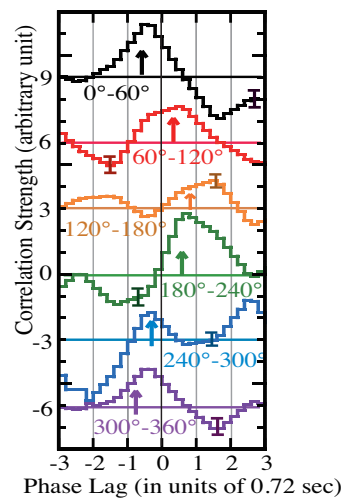

(b)

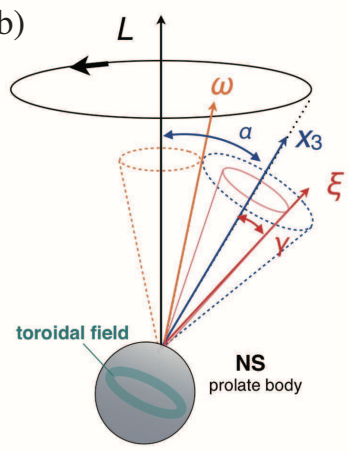

FIG. 3: (a) Cross correlation (interpolated and shown with offsets), in arbitrary unit, of the 2009 HXD profiles in Fig 1 (f), with those accumulated (without demodulation) in 6 phases of $T=55 \mathrm{ksec}$. Arrows indicate predictions by the best demodulation parameters. (b) An illustration of free precession of an axisymmetric rigid body [25, 26].

HXD data, which is rather sinusoidal (Fig. 3 a), could be due to the presence of a binary companion to the NS. From the observed values of $T$ and $A$, and the canonical NS mass of $1.4 M_{\odot}\left(M_{\odot}\right.$ being the Solar mass), the putative companion is estimated to have a mass of $0.12 M_{\odot} / \sin i$, where $i$ is the orbital inclination. Although the implied lower-limit mass of $\sim 0.1 M_{\odot}$ is broadly consistent with the optical $R$-band magnitude of $4 \mathrm{U} 0142+61, \sim 25 \mathrm{mag}$ [22], the optical emission, which is pulsed [23, 24], is likely to emerge from a vicinity of the NS, rather than from any companion star. Furthermore, the absence of the same modulation in the soft X-rays argues clearly against the binary interpretation.

As such, we consider that the pulse-phase modulation in the 2009 HXD data is more likely to reflect intrinsic dynamics of the NS in $4 \mathrm{U} 0142+61$, especifically, free precession of an axisymmetric rigid body which can occur without any external torque. In Fig. [3 (b) which illustrates an axisymmetric NS, let $\vec{L}$ be its angular momentum vector fixed to the inertial frame, and $\hat{x}_{3}$ a unit vector describing its axis of symmetry which we may identify with the dipolar magnetic axis. The star's asphericity is expressed by a quantity $\epsilon \equiv\left(I_{1}-I_{3}\right) / I_{3}$, where $I_{3}$ is the moment of inertia around $\hat{x}_{3}$, and $I_{1}$ that around axes orthogonal to $\hat{x}_{3}$. If $\epsilon \neq 0$, the $\hat{x}_{3}$ axis rotates around $\vec{L}$ at a constant period $P_{1}=2 \pi I_{1} / L$, with a constant "wobbling" angle $\alpha$ to $\vec{L}[25$ 27]. Likewise, the instantaneous rotation vector $\vec{\omega}$, co-planar with $\vec{L}$ and $\hat{x}_{3}$, rotates around $\vec{L}$, keeping a constant angle $\approx \epsilon \sin \alpha$ to $\vec{L}$.

Suppose that the NS emits photons through which we observe it, and express the direction of maximum photon emissivity by a unit vector $\hat{\xi}$ fixed to the NS. When $\hat{\xi}=\hat{x}_{3}$, the photons will reach us in periodic pulses with the period $P_{1}$. However, if $\hat{\xi}$ is tilted from $\hat{x}_{3}$ by a finite angle $\gamma$, then $\hat{\xi}$ will slowly rotate around $\hat{x}_{3}$ relative to the $\vec{L}-\vec{\omega}-\hat{x}_{3}$ plane, with a "slip period" 
$Q=P_{1} / \epsilon=\left[\left(1 / P_{3}-1 / P_{1}\right)\right]^{-1}$ where $P_{3} \equiv 2 \pi I_{3} / L$ 25 27]. The observed pulse arrival times then become subject to some jitter 26, and the effect can be approximated as a sinusoidal phase modulation in the regular pulsation, just as seen in Fig. 3(a).

The results from the 2009 HXD data thus allow an interpretation in terms of free precession of the NS with $T=Q$. The necessary condition of $\gamma \neq 0$ can be fulfilled if, e.g., the MF has multipole components [6, 11] $(\S 1)$. Due to some re-arrangement of the magnetic configuration, the hard X-ray emitting regions may wander around on the star, causing both $\gamma \sim 2 \pi a / P_{1} \sin (\alpha)$ and $\phi$ to change with time. The behavior of the two HXD datasets can be explained if the hard X-ray beam pattern was sharper with $\gamma \neq 0$ in 2009, while broader in 2007 with a smaller value of $\gamma$. The absence of the same modulation in the two XIS data sets can be explained if the soft X-ray emission comes form regions more symmetric $(\gamma \sim 0)$ around $\hat{x}_{3}$, and/or in a broader beam. However, other senarios remain; e.g., the putative motion of the hard X-ray source itself could produce red noise in the pulse phase, which mimics the 55 ksec periodicity.

If we employ the precession interpretation, the best-fit demodulation parameters yield $|\epsilon|=P_{1} / Q=P_{\text {hard }} / T=$ $1.6 \times 10^{-4}$. Although we cannot tell whether the object is prolate $(\epsilon>0)$ or oblate $(\epsilon<0)$, the former is more likely, because internal dissipation will increase $\alpha$ if $\epsilon>0$, while decrease if $\epsilon<0$. The strong X-ray pulses observed from nearly all magnetars, implying $\alpha \neq 0$, suggests $\epsilon>0$. Since an NS with $B_{\mathrm{t}} \gg B_{\mathrm{d}}$ would be deformed into a prolate shape with $\epsilon \sim+1.0 \times 10^{-4}\left(B_{\mathrm{t}} / 10^{12} \mathrm{~T}\right)^{2}$ [28 30 , the derived estimate of $\epsilon \sim 1 \times 10^{-4}$ can be explained by a toroidal magnetic field of $B_{\mathrm{t}} \sim 1 \times 10^{12} \mathrm{~T}$. It much exceeds the value of $B_{\mathrm{d}}=1.3 \times 10^{10} \mathrm{~T}$ derived from the pulse period and period derivative of this NS [20].

So far, there have been reports of possible detections of precession from accretion-powered pulsars [31], and fastrotating pulsars $32-35]$. However, the effects in the former objects should be regarded as forced precession, considering strong torque from the accreting matter. Similarly, the latter objects would not easily show free precession, since they must be deformed into oblate shapes under centrifugal force, and hence the precession would soon be damped [28], making the reports somewhat unconvincing [36]. In contrast, the present detection is considered more promising from the stability argument made above. (Decay in $\alpha$ due to emission of gravitational waves is expected to be insignificant [27].)

Supposing that our interpretation correctly account for the observation, several astrophysical implications follow. (1) It provides one of the first observational clues to $B_{t}$ inside NSs, which is much more difficult to estimate than $B_{\mathrm{d}}$ [1, 4]. (2) The relatively large value of $\epsilon$ supports the view that magnetars have $\left|B_{\mathrm{t}}\right|>\left|B_{\mathrm{d}}\right|[28,[29]$. (3) The differences between the soft and hard X-ray components suggests their distinct emission regions [13]. (4) Further studies of this object, and other similar ones, will provide valuable information on the NS interior [28, 30, 37], and prospects for gravitational-wave emission [27, 29, 38].

In summary, we suggest that; the NS in $4 \mathrm{U} 0142+61$ is deformed with $|\epsilon|=1.6 \times 10^{-4}$; the hard X-ray emission region moves, to some extent, on/around this NS; and the NS harbors an intense toroidal field of $B_{\mathrm{t}} \sim 10^{12} \mathrm{~T}$.

[1] R. N. Manchester and J. H. Taylor, Pulsars (W. H. Freeman, San Francisco: 1977).

[2] G. Chanmugam, Ann. Rev. Astr. Ap. 30, 143 (1992).

[3] A. Harding and D. Lai, Rep. Prog. Phys. 69, 2631 (2006).

[4] K. Makishima, T. Mihara, Y. Tanaka and F. Nagase, Astrophys. J. 525, 978 (1999).

[5] K. Makishima, Prog. Theor. Phys. Suppl. 151, 54 (2003).

[6] C. Thompson and R. C.Duncan, Mon. Not. R. Astr. Soc. 275, 255 (1995) .

[7] S. Mereghetti, Astron. Astrophys. Rev. 15, 225 (2008)

[8] S. Dall'Osso, S. N. Shore, and L. Stella, Mon. Not. R. Astr. Soc. 398, 1869 (2009).

[9] J. Braithwaite, , Mon. Not. R. Astr. Soc. 397, 763 (2009).

[10] N. Rea et al., Science 330, 944 (2010).

[11] A. Tiengo et al., Nature 500, 312 (2013).

[12] L. Kuiper, W. Hermsen, P. den Hartog and W. Collmar, Astrophys. J. 645, 556 (2006).

[13] T. Enoto et al., Astrophys. J. Lett. bf 722, L162 (2010).

[14] P. R. den Hartog et al., Astron. Ap. 451, 587 (2006).

[15] P. R. den Hartog et al., Astron. Ap. 489, 245 (2008).

[16] K. Mitsuda et al. Publ. Astr. Soc. Japan, 59, 1 (2007).

[17] K. Koyama et al., Publ. Astr. Soc. Japan 59, 23 (2007).

[18] T. Takahashi et al., Publ. Astr. Soc. Japan 59, 35 (2007).

[19] T. Enoto et al., Publ. Astr. Soc. Japan 63, 387 (2011).

[20] F. Gavriil and V. Kaspi, Astrophys. J. 567, 1067 (2002).

[21] K. T. Brazier, Mon. Not. R. Astr. Soc. 268, 709 (1994).

[22] M. Durant and M. H. van Kerkwijk, Astrophys. J. 652, 576 (2006).

[23] B. Kern and C. Martin, Nature 471, 527 (2002).

[24] V. Dhillon et al., Mon. Not.R.Astr. Soc. 363, 609 (2005).

[25] L. D. Landau and E. M. Lifshitz, Mechanics, Third Edition: Vol. 1 (Course of Theoretical Physics), Chapter IV (Butterworth-Heinemann; 1976).

[26] E. Butikov, European J. Physics 27, 1071 (2006).

[27] M. Maggiore, Gravitational Waves: Vol. 1: Theory and Experiments, §4.2.2 (Oxford University Press; 2007).

[28] C. Cutler, Phys. Review D 66, 084025 (2002).t

[29] L. Gualtieri, R. Ciolfi, and V. Ferrari, Class. Quantum Gravity 28, 114014 (2011).

[30] K. Glampedakis and D. I. Jones, Mon. Not. R. Astr. Soc. 405, L6 (2010).

[31] R. Staubert et al., Astron. Ap. 494, 1025 (2009).

[32] A. G. Lyne, R. S. Pritchard and G. Smith, Mon. Not. R. Astr. Soc. 233, 667 (1988).

[33] T. V. Shabanova, A. G. Lyne and J. O. Urama, Astrophys. J. 552, 231 (2001).

[34] A. E. Chukwude, A. Ubachukwu, and P. Okeke, Astron. Ap. 399, 231 (2003).

[35] F. Haberl et al., Astron. Ap. bf 451, L17 (2006).

[36] M. Ruderman and J. Gil, Astron. Ap. 460, L31 (2006).

[37] J. Lattimer and M. Prakash, Phys. Rep. 442, 109 (2007).

[38] J. Abadie et al., Astrophys. J. Lett. 734, L35 (2011). 